Group theoretic conditions for existence of robust relative homoclinic trajectories

Ashwin, Peter and Montaldi, James

2002

MIMS EPrint: 2006.247

Manchester Institute for Mathematical Sciences

School of Mathematics

The University of Manchester

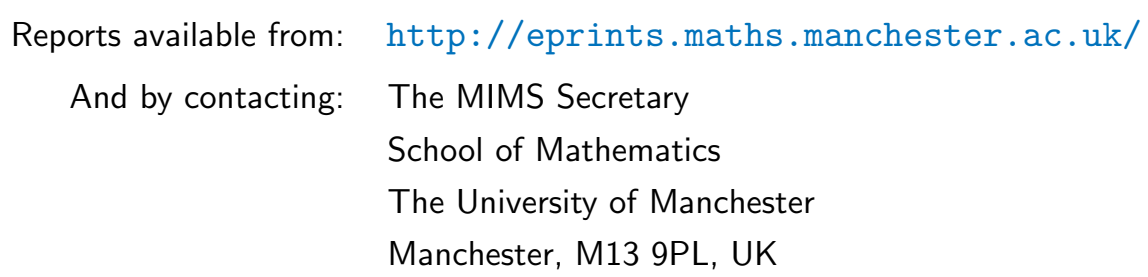

ISSN 1749-9097 
Math. Proc. Camb. Phil. Soc. (2002), 133, 125 (c) 2002 Cambridge Philosophical Society

\title{
Group theoretic conditions for existence of robust relative homoclinic trajectories
}

\author{
BY PETER ASHWIN \\ School of Mathematical Sciences, Laver Building, \\ University of Exeter, Exeter EX4 4QE. \\ AND JAMES MONTALDI \\ CNRS-INLN, 1361 route des Lucioles, 06560 Valbonne, France.
}

(Received 14 November 2000; revised 6 June 2001)

\begin{abstract}
We consider robust relative homoclinic trajectories (RHTs) for $G$-equivariant vector fields. We give some conditions on the group and representation that imply existence of equivariant vector fields with such trajectories. Using these results we show very simply that abelian groups cannot exhibit relative homoclinic trajectories. Examining a set of group theoretic conditions that imply existence of RHTs, we construct some new examples of robust relative homoclinic trajectories. We also classify RHTs of the dihedral and low order symmetric groups by means of their symmetries.
\end{abstract}

\section{Introduction}

Although homoclinic and heteroclinic cycles are not structurally stable to perturbations in typical systems, they do play a very important role in organizing the dynamics of trajectories that are nearby in phase and parameter space. In the presence of symmetries (or conserved quantities) it has been observed for many years that extra structure can force homoclinic orbits to be robust (i.e. they can persist under an open set of perturbations) and this gives their study in equivariant systems a special importance.

More precisely, suppose we have a flow generated by

$$
\dot{x}=v(x),
$$

where $x \in M$, a smooth manifold. Suppose that a compact Lie group $G$ acts smoothly on $M$ and that the flow generated by $v$ commutes with this action of $G$. We say $X_{0}=G \cdot x_{0}$ is a relative equilibrium if it is flow invariant, i.e. if $v\left(x_{0}\right) \in T\left(G \cdot x_{0}\right)$. A relative homoclinic trajectory (RHT) is a trajectory $\gamma(t)$ with $\gamma(t) \notin X_{0}$ but such that $d\left(\gamma(t), X_{0}\right) \rightarrow 0$ as $t \rightarrow \pm \infty$.

In the case of a finite group $G$ a relative equilibrium consists of a finite number of equilibrium points related to one another by the symmetry group, and a relative homoclinic trajectory consists of a trajectory that is homoclinic to or more generally heteroclinic between different points in the same relative equilibrium. More generally 
if $G$ is continuous, there will be a (usually infinite) number of connections between equilibria or invariant tori in $X_{0}$. We say a homoclinic trajectory is robust if it persists under an open set of sufficiently smooth perturbations.

Most studies of robust RHTs in equivariant systems have been either general results that assume the existence of certain structures (for example the conditions in [7] that imply attractivity of cycles) or they are specific examples where a vector field or normal form near bifurcation is studied in detail. In this paper we look at two general questions that are intimately related:

(i) How can we characterize the geometry of a relative homoclinic orbit?

(ii) How can we characterize group actions that allow structurally stable relative homoclinic trajectories?

After reviewing two motivating examples of Guckenheimer and Holmes [5] and of Kevrekidis et al. [6] in the remainder of this introduction, in Section 2 we introduce notation and describe the geometry of an RHT and an element in the group that we term the twist of an RHT. This is used in Proposition $2 \cdot 3$ to prove that a necessary condition for existence of a robust RHT is that the isotropies of trajectory, endpoints and twist are related in a certain way. As a trivial consequence, we deduce that one cannot have robust RHTs for abelian group actions.

In Section 3 we investigate the group theoretic conditions in Proposition 2.3 necessary for existence of robust RHTs. To this end, we introduce the notion of a homoclinic triple; this is a triple $(K, g, H)$ where $K$ and $H$ are subgroups and $g$ is a group element with certain properties that characterize the symmetries of a robust RHT. After demonstrating some useful invariance properties of homoclinic triples we classify in Theorem $3 \cdot 5$ the set of all possible homoclinic triples in the dihedral groups $\mathbf{D}_{n}$. This is followed by a discussion of homoclinic triples in the symmetric groups $S_{n}$ and also in some wreath product examples.

Section 4 adds extra hypotheses such that sufficient (but not necessary) conditions on a group action are found for robust RHTs; one of these conditions is group theoretic while the other concerns the geometry of the group representation. These conditions are used to construct a new example of an action of $S O(3)$ on a 16-dimensional vector space with robust RHTs and also a large class of finite groups that admit actions with robust RHTs. These include many with wreath product structure $[\mathbf{1}]$. The section ends with an example that explores the gap between the necessary and sufficient conditions for robust RHTs. Finally, Section 5 discusses extensions and consequences of this work.

For the remainder of this introductory section we recall two standard examples of systems and symmetry groups that permit robust relative homoclinic trajectories, partly for motivation, but also for later discussion.

\section{1·1. The Guckenheimer-Holmes robust RHT}

Consider the flow on $\mathbf{R}^{3}$ generated by

$$
\begin{aligned}
& \dot{x}_{1}=x_{1}\left(\lambda-x_{1}^{2}+b x_{2}^{2}+c x_{3}^{2}\right) \\
& \dot{x}_{2}=x_{2}\left(\lambda-x_{2}^{2}+b x_{3}^{2}+c x_{1}^{2}\right) \\
& \dot{x}_{3}=x_{3}\left(\lambda-x_{3}^{2}+b x_{1}^{2}+c x_{2}^{2}\right)
\end{aligned}
$$

where $b \neq c$ are real constants. This is equivariant under the group $G$ generated by $\kappa:\left(x_{1}, x_{2}, x_{3}\right) \mapsto\left(-x_{1}, x_{2}, x_{3}\right)$ and $\rho\left(x_{1}, x_{2}, x_{3}\right) \mapsto\left(x_{2}, x_{3}, x_{1}\right)$ (we could denote 
the group $G=\mathbf{Z}_{2}(\kappa) \succ \mathbf{Z}_{3}(\rho)$ using the wreath product notation, see [2], or $\mathbb{T}_{h}$ in the Schoenflies notation). For certain open sets of $(b, c)$ with $b c<0$, [5] observed and proved the existence of relative equilibria $G \cdot(1,0,0)$ connected by a relative homoclinic trajectory that is robust to any perturbation preserving the symmetry $G$, and in particular to the addition of higher order polynomial terms for the above vector field near $\lambda=0$. The equilibria in question have symmetry $\mathbf{Z}_{2}(\kappa) \times \mathbf{Z}_{2}\left(\rho \kappa \rho^{-1}\right)$ whereas the connections have symmetry $\mathbf{Z}_{2}(\kappa)$ (up to conjugation).

\section{1·2. The Kuramoto-Sivashinsky robust RHT}

As noticed by Kevrekidis, Nicolaenko and Scovel [6] the Kuramoto-Sivashinsky equation

$$
v_{t}+4 v_{x x x x}+\alpha\left(v_{x x}-v v_{x}\right)=0
$$

posed on the line $x \in[0,2 \pi]$ with periodic boundary conditions can display attracting RHTs for a certain range of parameter values. This system commutes with the group $O(2)$ of symmetries generated by translation $x \mapsto x+\theta$ modulo $2 \pi$, and reflection $x \mapsto 2 \pi-x$. This equation exhibits robust RHTs which can be seen in numerical simulations. In an $N$-mode truncated Fourier representation this can be seen by writing

$$
v(x, t)=\sum_{n=1}^{N}\left(y_{k}(t) \cos k x+y_{N+k}(t) \sin k x\right)
$$

and then for $\alpha \in(16.13,22.557)$ one can observe (in the truncated equations) RHTs connecting relative equilibria with $y_{k}=0$ for all $k=1, \ldots, N$ and $y_{N+k}=0$ for all odd $k=1,3,5, \ldots, N$. This RHT is simply the group orbit of an RHT contained in the fixed point subspace of the subgroup generated by $\kappa_{1}: x \rightarrow 2 \pi-x, \kappa_{2}: x \rightarrow \pi-x$ and $\rho: x \rightarrow x+\pi$ (all taken modulo $2 \pi$ on the domain). In this subspace the equilibria have symmetry $\mathbf{Z}_{2}\left(\kappa_{1}\right) \times \mathbf{Z}_{2}\left(\kappa_{2}\right)$ and the connections have symmetry $\mathbf{Z}_{2}\left(\kappa_{1}\right)$ only. These RHTs differ from the Guckenheimer-Holmes example in that any connections between a pair of equilibria occur in pairs.

\section{The geometry of relative homoclinic trajectories}

We consider relative homoclinic trajectories with reference to the flow on the orbit space $M / G$. Recall [3] that the orbit space $M / G$ has a natural stratification by orbit (isotropy) type, whose strata we denote by $(M / G)_{(H)}$, where $(H)$ is the conjugacy class of the subgroup $H$ of $G$.

We now recall some standard notation needed and then state the problem more precisely. The normalizer, $N_{G}(H)$, of $H$ in $G$ is the group of $g \in G$ such that $g H=H g$. $\operatorname{Fix}(H)$ is the fixed point space of $H$ in $M$, i.e. the set of $x \in M$ such that $h x=x$ for all $h \in H$. If $H$ is an isotropy subgroup then $g$ maps $\operatorname{Fix}(H)$ to itself if and only if $g \in N_{G}(H)$ [9]. For $g \in G$, we write $H^{g}$ to mean the conjugate subgroup by $g$, i.e. $H^{g}=g \mathrm{Hg}^{-1}$. We also use the notation $H<G$ to mean that $H$ is a subgroup of $G$.

Let $v$ be a $G$-equivariant vector field. The flow on $M$ descends to a flow on the orbit space $M / G$, induced by a stratified vector field $\bar{v}$. A relative homoclinic trajectory, or RHT, of the dynamics on $M$ is a trajectory whose image in $M / G$ is simply a homoclinic trajectory. For an RHT $\gamma(t)$ we denote its image in $M / G$ by $\bar{\gamma}(t)$. The $\alpha$ - and $\omega$-limit sets of the image $\bar{\gamma}(t)$ are then the same equilibrium. 


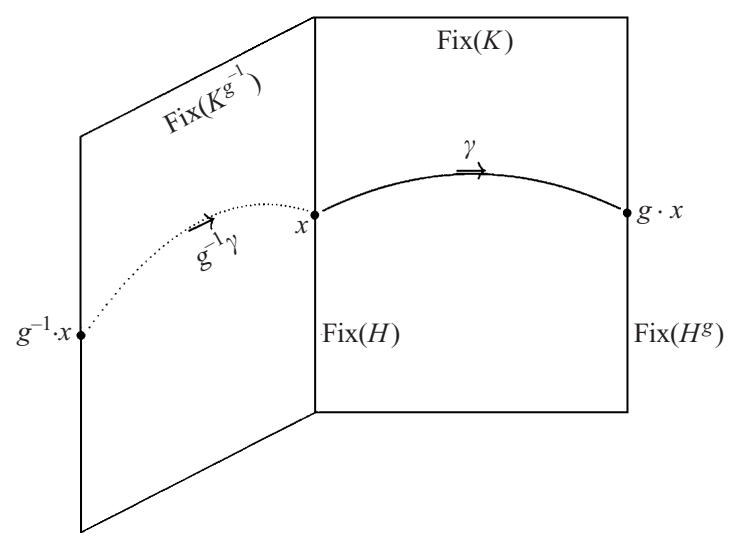

Fig. 1. Schematic diagram showing (part of) a relative homoclinic trajectory to a relative equilibrium $x$ in the phase space $M$. Note that there may be many connections to and from $x$ in the RHT; indeed there may also be many connections between $x$ and $g \cdot x$. In this case, the RHT has isotropy $K$ and twist $g$.

Let $\gamma(t)$ be an RHT. Then each point $\gamma(t)$ has the same isotropy subgroup, which we call the isotropy subgroup of $\gamma$ and denote by $K$.

The limit sets $\alpha(\gamma)$ and $\omega(\gamma)$ are closed, connected flow-invariant subsets of the relative equilibrium $\alpha(\bar{\gamma})=\omega(\bar{\gamma})$. These flow-invariant subsets also have an isotropy, and we denote the isotropy of $\alpha(\gamma)$ by $H$. By continuity we have that $K<H$.

If the group $G$ is finite, then $\alpha(\gamma)$ is an equilibrium point $x$, and $\omega(\gamma)=g \cdot x$, for some $g \in G$. This element $g$ we call the twist of the RHT. More properly, it is a twist, for it is only well-defined modulo $H$; that is, the twist is naturally an element of $G / H$, for if $y=g \cdot x$ then $y=g h \cdot x$ for all $h \in H$. The equilibrium point $\omega(\gamma)$ is of isotropy $H^{g}=g H g^{-1}$, and by continuity again $K<H^{g}$.

If on the other hand, $G$ is merely compact, $\alpha(\gamma)$ is of the form $T \cdot x$, for any $x \in \alpha(\gamma)$, and some torus $T<N_{G}(H)<G$ (not necessarily maximal). Moreover, $g \cdot x \in \omega(\gamma)$, for some $g \in G$, and $\omega(\gamma)=g T \cdot x=\left(g T g^{-1}\right) \cdot(g \cdot x)$. In either case, we call $g$ the twist of the RHT, and in this case it is well-defined modulo $(G / H) / T$. This twist tells how the homoclinic trajectory on $M / G$ lifts to a heteroclinic trajectory on $M$. Again the points of $\omega(\gamma)$ have isotropy $H^{g}$, and again $K<H^{g}$. Figure 1 schematically shows the setup within $M$.

Thus, associated to any Rнт, there are subgroups $K$ and $H$, and a twist $g \in G$ that is well-defined modulo $H$ in the finite, or modulo $H T$ in the compact case. We write this triple as $(K, g, H)$. If $\gamma$ is an RHT with triple $(K, g, H)$ then for $f \in G$ the RHT $f \cdot \gamma$ has triple $\left(K^{f}, g^{f}, H^{f}\right)$ as is readily checked. Note that $x$ will be a limit point for several distinct trajectories in the RHT, and indeed there may be more than one RHT between two given points $x$ and $y=g \cdot x$, as in the Kuramoto-Sivashinsky example.

Example $2 \cdot 1$. In the Guckenheimer-Holmes example in Section 1·1, the isotropy subgroup of the RHT is $K=\mathbf{Z}_{2}(\kappa)$, while that of the equilibrium $x=(0,0,1)$ is $H \simeq \mathbf{Z}_{2}(\kappa) \times \mathbf{Z}_{2}\left(\rho \kappa \rho^{-1}\right)$. The twist is then simply $g=\rho$. For an open set of coefficients, the RHT from $(0,0,1)$ tends to $(1,0,0)$, which is $g \cdot(0,0,1)$ for $g\left(x_{1}, x_{2}, x_{3}\right)=\left(x_{3}, x_{1}, x_{2}\right)$. Notice that $g \notin N_{G}(K)$. 
Example 2.2. For the Kuramoto-Sivashinsky example in Section $1 \cdot 2$ we have $K=$ $\mathbf{Z}_{2}\left(\kappa_{1}\right), H=\mathbf{Z}_{2}\left(\kappa_{1}\right) \times \mathbf{Z}_{2}\left(\kappa_{2}\right)$ and $g=\rho$. Hence $K \neq K^{g}$ but $H=H^{g}$; in fact $H$ is in a conjugacy class of its own because its normalizer is the whole group.

We consider only homoclinic orbits in $M$ that are homoclinic to normally hyperbolic relative equilibria, i.e. where the centre manifold $W^{c}(x)$ is contained in the group orbit $G \cdot x$ and so $\gamma \subset W^{u}(x) \cap W^{s}(g \cdot x)$. This is not a serious restriction because for generic equivariant vector fields, relative equilibria are normally hyperbolic (for example, see [3, lemma 3.1.10]).

The following result gives a simple necessary condition for an RHT to be robust.

Proposition 2.3. Let $\gamma$ be a robust $\mathrm{RHT}$ with isotropy type $K$ and twist $g$, and let $H$ be the isotropy of $\alpha(\gamma)$. Then

(i) $g H \cap N_{G}(K)=\varnothing$ and

(ii) $K<H \cap H^{g}$.

The first property implies both that $K \neq K^{g^{-1}}$ and that $H$ is a proper subgroup of $G$. The second property is equivalent to requiring that $H$ strictly contains both $K$ and $K^{g^{-1}}$.

Remark 2.4. The quotient $\left(H \cap H^{g}\right) / K$ tells us about the number of heteroclinic connections between $x$ and $g \cdot x$ within a given RHT, for if $\alpha(\gamma)=\alpha(f \cdot \gamma)=x$ and $\omega(\gamma)=\omega(f \cdot \gamma)=g \cdot x$ then $f \in H \cap H^{g}$. Moreover, $f \cdot \gamma=\gamma$ if and only if $f \in K$.

Proof (of Proposition 2.3). For (i) it is enough to show that $g \notin N_{G}(K)$, for $g$ is an arbitrary twist. Suppose first that $g \in N_{G}(K)$ and so $g$ maps $\operatorname{Fix}(K)$ to itself. Then all equilibria and connections lie within $M^{\prime}=\operatorname{Fix}(K)$ and so we consider the flow on $M^{\prime}$, which is equivariant under the action of $N_{G}(K) / K$. Note that the action of $N_{G}(K) / K$ is free at all points $\gamma(t)$.

Let $x \in \alpha(\gamma)$. Note that $g \cdot\left(W^{s}(x) \cap M^{\prime}\right)=W^{s}(g \cdot x) \cap M^{\prime}$ and so in particular $\operatorname{dim} W^{s}(x) \cap M^{\prime}=\operatorname{dim} W^{s}(g \cdot x) \cap M^{\prime}$. Since $\operatorname{dim}\left(W^{s}(g \cdot x) \cap M^{\prime}\right)+\operatorname{dim}\left(W^{u}(x) \cap M^{\prime}\right) \leqslant$ $\operatorname{dim} M^{\prime}$ any transverse intersection between $W^{u}(x)$ and $W^{s}(g \cdot x)$ must be trivial, i.e. the connection cannot be robust; this proves (i).

To see (ii), note that $g \cdot x \in \operatorname{Fix}(K)$ implies that $x \in g^{-1} \cdot \operatorname{Fix}(K)$ and so $x \in$ Fix $\left(K^{g-1}\right)$. By (i) $K \neq K^{g-1}$ and so the isotropy $H$ of $x$ contains both $K$ and $K^{g-1}$ but is not equal to either.

Corollary 2.5. If $G$ is abelian there can be no robust RHTs.

Proof. This is because if $G$ is abelian then $N_{G}(K)=G$ for all $K<G$ and so there is no twist satisfying Proposition 2·3(i).

In Proposition $3 \cdot 4$ we show similarly that there are no robust RHTs for actions of the dihedral group $\mathbf{D}_{n}$, unless $n$ is a multiple of 4 .

Remark 2.6. Although the previous corollary excludes the possibility of robust RHTS for abelian groups, one can find robust relative heteroclinic cycles for abelian groups. For example, one can break the cyclic symmetry of the GuckenheimerHolmes cycle by making $\lambda$ in (1.1) vary with index. This breaks the symmetry to the abelian group $\left(\mathbf{Z}_{2}\right)^{3}$ while leaving the same cycle robust. However, such perturbations will break the relative equilibria into three families of relative equilibria and 
the connections will no longer be homoclinic. Work of Melbourne et al. in particular gives general methods for locating robust relative heteroclinic cycles in terms of cycles in the isotropy lattice and conditions on the isotypic decompositions of certain isotropy subgroups [8].

\section{Classification of homoclinic triples}

Motivated by Proposition $2 \cdot 3$ we make the following definition. Given a group $G$, a homoclinic triple for $G$ is a triple $(K, g, H)$, where $K$ and $H$ are subgroups of $G$ and $g \in G$, satisfying

HT1: $g H \cap N_{G}(K)=\varnothing$ and

HT2: $K<H \cap H^{g}$.

Note that [HT2] is equivalent to assuming that $H>K \cup K^{g-1}$. Homoclinic triples in $G$ do not always give rise to robust RHTs of $G$-equivariant vector fields, though we show in Section 4 that with an additional hypothesis they do.

It is clear that if $(K, g, H)$ is a homoclinic triple for $G$, and $G<G^{\prime}$ then $(K, g, H)$ is a homoclinic triple for $G^{\prime}$.

Two homoclinic triples $(K, g, H)$ and $\left(K^{\prime}, g^{\prime}, H^{\prime}\right)$ are said to be conjugate if there is an element $f \in G$ such that $K^{\prime}=K^{f}, H^{\prime}=H^{f}$ and $g^{\prime}=g^{f}=f g f^{-1}$. Moreover, if there is a vector field such that the first is the triple associated to an RHT $\gamma$ then the second is the triple associated to the RHT $f \cdot \gamma$, for the same vector field.

A further equivalence can be given by the relation $(K, g, H) \sim\left(K, g^{-1}, H^{g}\right)$. Indeed, it is easy to see that if a vector field $v$ gives a robust RHT with triple $(K, g, H)$, then the opposite vector field $-v$ gives a robust RHT with triple $\left(K, g^{-1}, H^{g}\right)$.

What seems less obvious is the following fact, whose relationship with the dynamics is not clear.

Proposition 3·1. If $(K, g, H)$ is a homoclinic triple, then so is $(K, \nu g, H)$ for all $\nu \in N_{G}(K)$.

Proof. Suppose $(K, g, H)$ is a homoclinic triple, and let $g^{\prime}=\nu g$ for $\nu \in N_{G}(K)$. Then, firstly, if $g H \cap N_{G}(K)=\varnothing$ then $\nu g H \cap \nu N_{G}(K)=\varnothing$, but $\nu N_{G}(K)=N_{G}(K)$, and secondly we have $H>K$ and since $K^{g-1}=K^{(\nu g)^{-1}}$ we have $H>K^{g^{\prime-1}}$ as required.

It is clear that if $(K, g, H)$ is a homoclinic triple for $G$, and $G_{1}<G$ is a subgroup containing $g$, then $\left(K \cap G_{1}, g, H \cap G_{1}\right)$ is a homoclinic triple for $G_{1}$. More generally, the property of being a homoclinic triple is preserved under pull-back:

Proposition 3י2. Let $\phi: G_{1} \rightarrow G_{2}$ be a homomorphism, and let $\left(K_{2}, g_{2}, H_{2}\right)$ be a homoclinic triple in $G_{2}$, with $g_{2} \in \operatorname{Im}(\phi)$. Define $K_{1}=\phi^{-1}\left(K_{2}\right), H_{1}=\phi^{-1}\left(H_{2}\right)$ and let $g_{1} \in \phi^{-1}\left(g_{2}\right)$. Then $\left(K_{1}, g_{1}, H_{1}\right)$ is a homoclinic triple in $G_{1}$.

Proof. It is easy to check that if $g_{1} H_{1} \cap N_{G_{1}}\left(K_{1}\right) \neq \varnothing$ then the image under $\phi$ of any element in this intersection belongs to $g_{2} H_{2} \cap N_{G_{2}}\left(K_{2}\right)$ which by hypothesis is empty, thus establishing [HT1]. Similarly, [HT2] also follows simply by applying $\phi^{-1}$.

A useful result for classifying homoclinic triples is the following: 
Proposition 3.3. Suppose $(K, g, H)$ is a homoclinic triple. Then so is $\left(K, g, H_{0}\right)$, where $H_{0}$ is the group generated by $K$ and $K^{g^{-1}}$.

Proof. It is clear that [HT1] is still satisfied while [HT2] is satisfied by construction.

Such triples $\left(K, g, H_{0}\right)$ are called minimal homoclinic triples, since necessarily $H_{0}<H$.

The aim is to classify such minimal homoclinic triples up to the equivalence relation generated by conjugacy and the equivalence of Proposition 3.1. The procedure is as follows. Fix a subgroup $K$, and choose a distinct conjugate $K^{\prime}=K^{g^{-1}}$, for some $g$ which is well-defined modulo $N_{G}(K)$ (as in the proposition above). Let $H=H_{0}$ be the group generated by $K$ and $K^{\prime}$ (the smallest possible group satisfying [HT2']), and finally check the remaining criterion [HT1]. In simple cases - such as small permutation groups - the checking can be carried out by Maple.

Having found the smallest possible $H_{0}$ for a given pair $(K, g)$ one can then consider enlarging $H$ until [HT1] is no longer satisfied.

The smallest non-abelian group is the symmetric group $S_{3}=\mathbf{D}_{3}$, but there is only one non-normal subgroup of $S_{3}$ up to conjugation, namely a $\mathbf{Z}_{2}$, and for this subgroup there is no possible twist, as is readily checked.

One of the next smallest non-abelian groups is the symmetry group $\mathbf{D}_{4}$ of the square, and for this there are homoclinic triples, as found in the KuramotoSivashinsky example. In fact there are two homoclinic triples, which are not conjugate, though they are equivalent under an outer automorphism of the group.

Another non-abelian example of the same size is the eight-element quaternion group $Q=\{ \pm 1, \pm i, \pm j, \pm k\}$ where $i^{2}=j^{2}=k^{2}=i j k=-1$. The only non-trivial groups $K$ and $H$ such that $G>H>K$ are non-trivial containments have $K=\{ \pm 1\}$. As $N_{G}(K)=G$ [HT1] cannot be satisfied and $Q$ supports no homoclinic triples.

In each of the tables of the sections that follow, the final column states whether $K$ is a normal subgroup of both $H$ and $H^{g}$,or equivalently whether $H$ is contained in the normalizers of both $K$ and $K^{g^{-1}}$. This is a condition we will use in the existence theorem of Section 4.

\subsection{Dihedral groups}

Denoted $\mathbf{D}_{n}$, these are the symmetry groups of the regular polygons. In order to determine homoclinic triples in $\mathbf{D}_{n}$ we introduce some notation, and recall a few basic properties of $\mathbf{D}_{n}$. Let $\rho$ be the rotation by $2 \pi / n$, and $\kappa$ a reflection, so that $\mathbf{D}_{n}=\langle\kappa, \rho\rangle$, and $\rho \kappa=\kappa \rho^{-1}$. The elements of $\mathbf{D}_{n}$ can then be written as

$$
\left\{1, \rho, \rho^{2}, \ldots, \rho^{n-1}, \kappa, \kappa \rho, \ldots, \kappa \rho^{n-1}\right\} .
$$

Any subgroup of the cyclic subgroup $\mathbf{Z}_{n}$ of $\mathbf{D}_{n}$, is normal in $\mathbf{D}_{n}$, so candidates for the symmetry group $K$ of a robust RHT must contain a reflection. If $n$ is even then there are two distinct conjugacy classes of reflection, given by $\kappa$ and $\kappa \rho$. More generally, $\kappa \rho^{r} \sim \kappa \rho^{s}$ if and only if $r$ and $s$ are of the same parity modulo $n$. 


\begin{tabular}{llllll}
$G=\mathbf{D}_{4}$ & & & & \\
\hline$K$ & generators of $K$ & twist, $g$ & $H$ & generators of $H$ & $K \triangleleft H, H^{g}$ \\
\hline $\mathbf{Z}_{2}$ & $\kappa$ & $\rho$ & $\mathbf{D}_{2}$ & $\kappa, \rho^{2}$ & Yes \\
$\mathbf{Z}_{2}$ & $\kappa \rho$ & $\rho$ & $\mathbf{D}_{2}^{\prime}$ & $\kappa \rho, \rho^{2}$ & Yes \\
\hline
\end{tabular}

One of these two homoclinic triples, say with $K$ generated by $\kappa$, occurs in the Kuramoto-Sivashinsky system. The other one does not as $\mathbf{D}_{2}^{\prime}$ is not an isotropy subgroup. It should be pointed out that the two rows are equivalent under an outer automorphism of the group but they are not conjugate, which is why both are included.

$G=\mathbf{D}_{8} \quad$ This group contains $\mathbf{D}_{4}$ so two cases follow from those of $\mathbf{D}_{4}$, namely those with twist $\rho^{2}$.

\begin{tabular}{llllll}
\hline$K$ & generators of $K$ & twist, $g$ & $H$ & generators of $H$ & $K \triangleleft H, H^{g}$ \\
\hline $\mathbf{Z}_{2}$ & $\kappa$ & $\rho$ & $\mathbf{D}_{4}$ & $\kappa, \rho^{2}$ & No \\
$\mathbf{Z}_{2}$ & $\kappa \rho$ & $\rho$ & $\mathbf{D}_{4}$ & $\kappa \rho, \rho^{2}$ & No \\
$\mathbf{Z}_{2}$ & $\kappa$ & $\rho^{2}$ & $\mathbf{D}_{2}$ & $\kappa, \rho^{4}$ & Yes \\
$\mathbf{Z}_{2}$ & $\kappa \rho$ & $\rho^{2}$ & $\mathbf{D}_{2}$ & $\kappa \rho, \rho^{4}$ & Yes \\
$\mathbf{D}_{2}$ & $\kappa, \rho^{4}$ & $\rho$ & $\mathbf{D}_{4}$ & $\kappa, \rho^{2}$ & Yes \\
$\mathbf{D}_{2}$ & $\kappa \rho, \rho^{4}$ & $\rho$ & $\mathbf{D}_{4}$ & $\kappa \rho, \rho^{2}$ & Yes \\
\hline
\end{tabular}

The first two lines are equivalent under an outer automorphism of $G$, as are each of the next pair and the final pair.

Proposition 3.4. $\mathbf{D}_{n}$ contains homoclinic triples if and only if $n$ is a multiple of 4.

In particular, by Proposition $2 \cdot 3$ there are no robust RHTs in systems with dihedral symmetry $\mathbf{D}_{n}$ unless $n$ is a multiple of 4 .

Proof. First, if $n$ is a multiple of 4 , then $\mathbf{D}_{4}<\mathbf{D}_{n}$, so the result follows from finding a homoclinic triple for $\mathbf{D}_{4}$. This is given in the table above.

For the converse, let $K<G$ be a non-normal subgroup, and suppose $(K, g, H)$ is a homoclinic triple. As argued above, we may assume $\kappa \in K$. Without loss of generality we can take the twist to be $g=\rho^{r}$ for some $r$, for by Proposition $3 \cdot 1\left(K, p^{r}, H\right)$ is a homoclinic triple if and only if $\left(K, \kappa \rho^{r}, H\right)$ is since $\kappa \in K<N_{G}(K)$. Then, $K^{g^{-1}}$ contains $\rho^{-r} \kappa \rho^{r}=\kappa \rho^{2 r}$. It follows in particular that $h=\rho^{2 r} \in H$.

Suppose first that $n$ is odd, and put $a=(n-1) / 2$. Then $g h^{a}=\rho^{r(2 a+1)}=\rho^{r n}=1 \epsilon$ $N_{G}(K)$ contradicting the hypothesis that $(K, g, H)$ is a homoclinic triple.

Now suppose $n=2 p$, with $p$ odd. Then $\rho^{p}$ (rotation by $\pi$ in $\mathbf{D}_{n}$ ) is in the centre of $\mathbf{D}_{n}$ so that it belongs to $N_{G}(K)$ for any subgroup $K$. Now repeat the argument above but with $a=(p-1) / 2$, and one finds that $g h^{a}=\rho^{p} \in N_{G}(K)$.

We now give a complete classification of homoclinic triples for subgroups of $\mathbf{D}_{4 n}$. For uniformity of notation, we write $\mathbf{Z}_{2}=\mathbf{D}_{1}$ and denote by $t(r)$ the multiplicity of 2 in the prime decomposition of the integer $r$. 
Theorem 3.5. Let $G=\mathbf{D}_{4 n}$. Up to equivalence, the only homoclinic triples are of the form $(K, g, H)=\left(\mathbf{D}_{q}, \rho^{r}, \mathbf{D}_{p}\right)$, satisfying:

(i) $q \mid n$;

(ii) $t(r) \leqslant t(n / q)$;

(iii) $q|p, q \neq p, p|(2 n)$ and $r \equiv(2 n / p) \bmod (4 n / p)$.

Moreover all such triples are homoclinic triples.

Proof. As has already been pointed out, homoclinic triples cannot have $K=\mathbf{Z}_{q}$ for these are normal subgroups of $G=\mathbf{D}_{n}$. Thus we can suppose $K=\mathbf{D}_{q}$ for some $q \geqslant 1$ which necessarily divides $4 n$. Write $s=4 n / q$, and let $\kappa$ be a reflection in $K$. Thus $\mathbf{D}_{q}=\left\langle\kappa, \rho^{s}\right\rangle$. The normalizer $N_{G}(K)$ is $\mathbf{D}_{q^{\prime}}$, where $q^{\prime}=q$ if $s$ is odd, and $q^{\prime}=2 q$ if $s$ is even. Thus, $N_{G}(K)=\left\langle\kappa, \rho^{s^{\prime}}\right\rangle$, where $q^{\prime} s^{\prime}=4 n$. If $K=\langle\kappa\rangle$ (i.e., if $q=1$ ) then $s=4 n$ and $s^{\prime}=2 n$.

Without loss of generality, we can assume the twist $g=\rho^{r}$ for some $r$, for the alternative form is $\kappa \rho^{r}$, but the two are equivalent by Proposition 3.1. Furthermore, by the same equivalence, we can choose $0<r<s^{\prime}$.

Let $H_{0}$ be the subgroup generated by $K$ and $K^{g^{-1}}$. Then

$$
H_{0}=\left\langle\kappa, \rho^{2 r}, \rho^{s}\right\rangle,
$$

and

$$
g H_{0}=\left\{\rho^{r+k 2 r+\ell s}, \kappa \rho^{-r+k 2 r+\ell s} \mid k, \ell \in \mathbf{Z}\right\},
$$

so that $g H_{0} \cap N_{G}(K) \neq \varnothing$ if and only if there are integers $j, k, \ell$ such that

$$
\rho^{r+j s+2 k r}=\rho^{\ell s^{\prime}} \quad \text { or } \quad \kappa \rho^{-r+j s+2 k r}=\kappa \rho^{\ell s^{\prime}} .
$$

This is equivalent to there being integers $k, \ell$ such that

$$
(2 k+1) r=\ell s^{\prime},
$$

If $q$ does not divide $n$ then $s^{\prime}$ is odd and there are always solutions to this equation (for all $r$ ), so establishing (i).

Now suppose $q \mid n$, so that $s^{\prime}$ is even. Then (3.1) has solutions if and only if $t(r) \geqslant t\left(s^{\prime}\right)$ so establishing (ii).

Finally, let $H=\mathbf{D}_{p}=\left\langle\kappa, \rho^{a}\right\rangle$, for some $a$ which must divide both $s=2 s^{\prime}$ and $2 r$ (so that $H$ contains both $K$ and $K^{g^{-1}}$ ). If $a$ divides $r$ then $g \in H$ which is not possible. Thus $a$ is even since it divides $2 r$, and $r \equiv(a / 2) \bmod a$.

In this case $g H \cap N_{G}(K) \neq \varnothing$ if and only if there are integers $k, \ell$ such that

$$
r+k a=\ell s^{\prime} .
$$

Since $a \mid s$ and $s=2 s^{\prime}$ we have either $s^{\prime}=0 \bmod a$ or $s^{\prime} \equiv(a / 2) \bmod a$. In the first case there are no solutions to the equation, while in the second there are solutions, so establishing (iii).

If $K=\mathbf{D}_{q}$ then, up to equivalence, the only case where $K$ and $K^{g^{-1}}$ are normal subgroups of $H$ is $H=\mathbf{D}_{2 q}$ and $g=\rho^{n / q}$.

\subsection{Symmetric groups}

$G=S_{3} \simeq \mathbf{D}_{3} \quad$ We have already proved that this group has no homoclinic triples (Proposition 3.4). 
$G=S_{4} \simeq \mathbb{T}_{d}$ The only inequivalent homoclinic triples are given in the following table:

\begin{tabular}{|c|c|c|c|c|c|}
\hline$K$ & generators of $K$ & twist, $g$ & $H$ & generators of $H$ & $K \triangleleft H, H^{g}$ \\
\hline $\mathbf{Z}_{2}$ & $\left(\begin{array}{ll}1 & 2\end{array}\right)$ & $\left(\begin{array}{lll}1 & 3\end{array}\right)(24)$ & $\mathbf{D}_{2}$ & $\left(\begin{array}{l}1 \\
2\end{array}\right),(34)$ & Yes \\
\hline $\mathbf{Z}_{2}$ & $\left(\begin{array}{ll}1 & 2\end{array}\right)(34)$ & $\left(\begin{array}{ll}1 & 3\end{array}\right)$ & $V_{4}$ & $\left(\begin{array}{lll}1 & 2\end{array}\right)(34),\left(\begin{array}{lll}1 & 4\end{array}\right)\left(\begin{array}{ll}2 & 3\end{array}\right)$ & Yes \\
\hline
\end{tabular}

$G=S_{5} \quad$ The only homoclinic triples appear to be those that come from the inclusion $S_{4}<S_{5}$.

$G=S_{6} \quad$ This list is by no means expected to be exhaustive; there are many different classes of subgroup of $S_{6}$.

\begin{tabular}{|c|c|c|c|c|c|}
\hline$K$ & generators of $K$ & twist, $g$ & $H$ & generators of $H$ & $K \triangleleft H, H^{g}$ \\
\hline $\mathbf{Z}_{2}$ & $\left(\begin{array}{ll}1 & 2\end{array}\right)$ & $\left(\begin{array}{llll}1 & 3\end{array}\right)(2 \quad 4)$ & $\mathbf{D}_{2}$ & $(12),(34)$ & Yes \\
\hline $\mathbf{Z}_{2}$ & $(12)(34)$ & $\left(\begin{array}{lll}1 & 3\end{array}\right)$ & $V_{4}$ & $\left(\begin{array}{lll}1 & 2\end{array}\right)(34),\left(\begin{array}{lll}1 & 3\end{array}\right)(24)$ & Yes \\
\hline $\mathbf{Z}_{2}$ & $\left(\begin{array}{lll}1 & 2\end{array}\right)(34)$ & $\left(\begin{array}{llll}1 & 5\end{array}\right)\left(\begin{array}{lll}2 & 6 & 3\end{array}\right)$ & $\mathbf{D}_{4}$ & $\left(\begin{array}{lll}1 & 2\end{array}\right)(34),\left(\begin{array}{ll}2 & 4\end{array}\right)\left(\begin{array}{ll}5 & 6\end{array}\right)$ & No \\
\hline $\mathbf{Z}_{2}^{2}$ & $\left(\begin{array}{ll}1 & 2\end{array}\right),(34)$ & $(15)(26)$ & $\mathbf{Z}_{2}^{3}$ & $\left(\begin{array}{l}1 \\
2\end{array}\right),(34),(56)$ & Yes \\
\hline $\mathbf{Z}_{2}^{2}$ & $\left(\begin{array}{ll}1 & 2\end{array}\right),(34)$ & $(135)(26)$ & $\mathbf{Z}_{2} \times S_{4}$ & $(12),(34),(35),(36)$ & No \\
\hline $\mathbf{C}_{3}$ & $\left(\begin{array}{lll}1 & 2 & 3\end{array}\right)$ & $(14)(25)(36)$ & $\mathbf{Z}_{3}^{2}$ & $\left(\begin{array}{lll}1 & 2 & 3\end{array}\right),\left(\begin{array}{lll}4 & 5 & 6\end{array}\right)$ & Yes \\
\hline $\mathbf{C}_{6}$ & 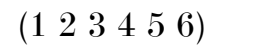 & $(24)$ & $W$ & $(123456),\left(\begin{array}{l}123654) \\
(1)\end{array}\right.$ & No \\
\hline $\mathbf{D}_{6}$ & $\begin{array}{l}\left(\begin{array}{lll}1 & 6\end{array}\right)\left(\begin{array}{lll}2 & 5\end{array}\right)\left(\begin{array}{ll}3 & 4\end{array}\right) \\
\left(\begin{array}{llllll}1 & 2 & 3 & 4 & 5 & 6\end{array}\right)\end{array}$ & $(24)$ & $W$ & $(123456),\left(\begin{array}{l}123 \\
(1254)\end{array}\right.$ & No \\
\hline $\mathbf{Z}_{6}$ & 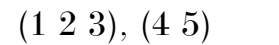 & $(14)(2536)$ & $S_{3}^{2}$ & $\left(\begin{array}{l}1 \\
2\end{array}\right),(13),(45),(46)$ & No \\
\hline$S_{3}$ & $\left(\begin{array}{lll}1 & 2\end{array}\right),\left(\begin{array}{lll}1 & 3\end{array}\right)$ & $(14)(25)(36)$ & $S_{3}^{2}$ & $\left(\begin{array}{l}1 \\
2\end{array}\right),(13),(45),(46)$ & Yes \\
\hline
\end{tabular}

The group $W$ has order 36 and has the two generators as shown.

\subsection{Wreath products}

Wreath products are the natural form of symmetry group occurring in systems of coupled cells. They are groups of the form $G=\mathscr{L} \imath \mathscr{G}$ where $\mathscr{G}<S_{n}$ is a subgroup of a permutation group and $\mathscr{L}$ is a non-trivial compact group; see for example [1, 2]. Here we consider two specific examples.

$G=\mathbf{Z}_{2} \curlywedge \mathbf{Z}_{3} \simeq A_{4} \times \mathbf{Z}_{2} \simeq \mathbb{T}_{h}$ This is the group occurring in the GuckenheimerHolmes example, see Section 1.1. We write $\rho$ for the generator of $\mathbf{Z}_{3}$ and $\kappa_{j}$ for the generator of the $j$ th copy of $\mathbf{Z}_{2}$.

\begin{tabular}{llllll}
\hline$K$ & generators of $K$ & twist, $g$ & $H$ & generators of $H$ & $K \triangleleft H, H^{g}$ \\
\hline $\mathbf{Z}_{2}$ & $\kappa_{1}$ & $\rho$ & $\mathbf{D}_{2}$ & $\kappa_{1}, \kappa_{2}$ & Yes \\
$\mathbf{Z}_{2}$ & $\kappa_{1} \kappa_{2}$ & $\rho$ & $\mathbf{D}_{2}$ & $\kappa_{1} \kappa_{2}, \kappa_{1} \kappa_{3}$ & Yes \\
$\mathbf{D}_{2}$ & $\kappa_{1}, \kappa_{2}$ & $\rho$ & $\mathbf{Z}_{2}^{3}$ & $\kappa_{1}, \kappa_{2}, \kappa_{3}$ & Yes \\
$\mathbf{Z}_{2}^{2}$ & $\kappa_{1}, \kappa_{2} \kappa_{3}$ & $\rho$ & $\mathbf{Z}_{2}^{3}$ & $\kappa_{1}, \kappa_{2}, \kappa_{3}$ & Yes \\
\hline
\end{tabular}

The first row is the case occurring in the Guckenheimer-Holmes example. Observe that this is only one of a number of possible homoclinic triples. 
$G=\mathbf{Z}_{2} \zeta S_{3} \quad$ This is the octahedral group $\mathbb{O}_{h}$. Write $\rho=\left(\begin{array}{lll}1 & 2 & 3\end{array}\right)$ and $\sigma=\left(\begin{array}{ll}1 & 2\end{array}\right)$ in $S_{3}$ and let $\kappa_{j}$ be the generator of $\mathbf{Z}_{2}$ in the $j$ th position.

\begin{tabular}{llllll}
\hline$K$ & generators of $K$ & twist, $g$ & $H$ & generators of $H$ & $K \triangleleft H, H^{g}$ \\
\hline $\mathbf{Z}_{2}$ & $\kappa_{1}$ & $\sigma$ & $\mathbf{Z}_{2}^{2}$ & $\kappa_{1}, \kappa_{2}$ & Yes \\
$\mathbf{Z}_{2}$ & $\kappa_{1} \kappa_{2}$ & $\sigma$ & $\mathbf{Z}_{2}^{2}$ & $\kappa_{1} \kappa_{2}, \kappa_{1} \kappa_{3}$ & Yes \\
$\mathbf{Z}_{2}$ & $\sigma$ & $\kappa_{1}$ & $\mathbf{D}_{2}$ & $\sigma, \kappa_{1} \kappa_{2}$ & Yes \\
$\mathbf{Z}_{2}$ & $\sigma \kappa_{3}$ & $\kappa_{1}$ & $\mathbf{D}_{2}$ & $\sigma \kappa_{3}, \kappa_{1} \kappa_{2} \sigma$ & Yes \\
$\mathbf{Z}_{2}^{2}$ & $\sigma, \kappa_{3}$ & $\kappa_{1}$ & $\mathbf{Z}_{2}^{3}$ & $\sigma, \kappa_{1} \kappa_{2}, \kappa_{3}$ & Yes \\
$\mathbf{Z}_{2}^{2}$ & $\kappa_{1}, \kappa_{2}$ & $\rho$ & $\mathbf{Z}_{2}^{3}$ & $\kappa_{1}, \kappa_{2}, \kappa_{3}$ & Yes \\
\hline
\end{tabular}

Example 3.6. It is easy to extend some of these to the general case $G=\mathscr{L}\{\mathscr{G}$. We set $K$ to be a subgroup consisting of $\mathscr{L}$ in only one component, say $\mathscr{L}_{1}$ and identity elsewhere. If we take $g \in \mathscr{G}$ which takes cell 1 to say, cell 2 (e.g., $g=\left(\begin{array}{l}12 \\ 2\end{array}\right) \in S_{n}$ corresponding to $\sigma$ in the table above or $g=\left(\begin{array}{lll}1 & 2 & 3\end{array}\right)$ corresponding to $\rho$ in the previous table) then $N_{G}(K)=\mathscr{L}_{1} \times\left(\mathscr{L} \imath \mathscr{G}^{\prime}\right)$ for some subgroup $\mathscr{G}^{\prime}$ of $\mathscr{G}$, and $K^{g^{-1}}=\mathscr{L}_{2}$. Let $H=\mathscr{L}_{1} \times \mathscr{L}_{2}$ (or any other subgroup of $N_{G}(K)$ containing $\mathscr{L}_{1} \times \mathscr{L}_{2}$ ) then in addition to being a homoclinic triple, one also finds that $K \triangleleft H, H^{g}$.

\section{Construction of robust RHTs}

Proposition 2.3 gives group-theoretic conditions on the twist of an RHT necessary for it to be robust. We now give sufficient conditions that allow construction of robust RHTS.

Theorem 4·1. Let $G$ be a compact Lie group acting on $M$. Suppose $K$ and $H$ are isotropy subgroups of this action and $(K, g, H)$ is a homoclinic triple satisfying the two further conditions (one 'local' the other 'global'):

HTL $H<N_{G}(K) \cap N_{G}\left(K^{g^{-1}}\right)$,

HTG there is a point $x \in M$ with isotropy $H$ and a continuous path of points with isotropy $K$ that joins $x$ to $g \cdot x \in \operatorname{Fix}\left(H^{g}\right)$.

Then there exists a non-empty open set of equivariant vector fields on $M$ with a robust RHT of isotropy $K$, based on a point in $\operatorname{Fix}(H)$ and with twist $g$.

Proof. Note that if $K$ is an isotropy subgroup then so is $K^{g}$ and by the fact that $(K, g, H)$ is a homoclinic triple, $K$ and $K^{g^{-1}}$ are distinct isotropy subgroups and so have distinct fixed point subspaces. Let $X_{1}$ be the non-empty open set of equivariant vector fields on $M$ for which there is a normally hyperbolic (relative) equilibrium point $x$ with isotropy $H$.

Consider the action of $H$ on $T_{x} M$, and consider the three subspaces $T_{H}=$ $\operatorname{Fix}\left(H, T_{x} M\right), T_{K}=\operatorname{Fix}\left(K, T_{x} M\right)$ and $T_{K}^{\prime}=\operatorname{Fix}\left(K^{g^{-1}}, T_{x} M\right)$. By [HTL] these are each $H$-invariant subspaces. It follows that there is an $H$-invariant decomposition of $T_{x} M$ as

$$
T_{x} M=T_{H} \oplus T_{0} \oplus T_{1} \oplus T_{2} \oplus W
$$


where

$$
\begin{aligned}
T_{H} \oplus T_{0} & =T_{K} \cap T_{K}^{\prime} \\
T_{H} \oplus T_{0} \oplus T_{1} & =T_{K} \\
T_{H} \oplus T_{0} \oplus T_{2} & =T_{K}^{\prime} .
\end{aligned}
$$

The fact that $T_{H} \subset T_{K} \cap T_{K}^{\prime}$ follows from the homoclinic triple property. $T_{1}$ and $T_{2}$ are of course of the same dimension. We treat explicitly the case where $G$ is finite; the general case can be deduced by intersecting the above decomposition with a local slice to the group orbit through $x$.

While the linearization $u \mapsto L u$ of the vector field at $x$ leaves invariant each of $T_{H}, T_{K}, T_{K}^{\prime}$ and $T_{K} \cap T_{K}^{\prime}$, it does not necessarily leave invariant the entire decomposition. However, there are eigenvalues associated to each subspace. For example since $T_{H}$ and $T_{H} \oplus T_{0}$ are invariant, it follows that the linear vector field descends to a vector field on $\left(T_{H} \oplus T_{0}\right) / T_{H} \simeq T_{0}$ whose eigenvalues we call $\sigma_{0}(L)$. Similar constructions define $\sigma_{1}(L), \sigma_{2}(L)$ etc.

Define the class $X_{2}$ of vector fields in $X_{1}$ for which

$$
m_{1}^{+}+m_{2}^{-}>\operatorname{dim} T_{1}
$$

where $m_{1}^{+}$is the number of eigenvalues in $\sigma_{1}(L)$ with positive real part, counting multiplicity, and similarly $m_{2}^{-}$counts, with multiplicity, the number of eigenvalues in $\sigma_{2}(L)$ with negative real part. This class $X_{2}$ is clearly open in $X_{1}$ and non-empty. Furthermore, the members of this class satisfy

$$
\left(W^{u}(x) \cap \operatorname{Fix}(K)\right)+\operatorname{dim}\left(W^{s}(g \cdot x) \cap \operatorname{Fix}(K)\right)>\operatorname{dim} \operatorname{Fix}(K) .
$$

This is because $g \cdot\left(W^{s}(x) \cap \operatorname{Fix}\left(K^{g^{-1}}\right)\right)=W^{s}(g \cdot x) \cap \operatorname{Fix}(K)$.

Now consider any continuous path $\gamma(t)$ with finite are length, connecting $x$ to $g \cdot x$ in $\operatorname{Fix}(K)$ such that $\gamma(t)$ has isotropy $K$. Given a tubular neighbourhood $N$ of $\gamma$ such that $g(N)$ does not intersect $N$, there is an open set $X_{3} \subset X_{2}$ of vector fields such that $W^{u}(x)$ and $W^{s}(g \cdot x)$ intersect on a path within $N$.

To see that the set $X_{3}$ is not empty, we need to construct the heteroclinic connection, and to do this we pass to the orbit space $M / G$. We write $\bar{x}$ for the image of $x$ in the orbit space, and similarly $\overline{\operatorname{Fix}(K)}$ etc. Consider a vector field in $X_{2}$ and its image on the orbit space, and restrict it to a neighbourhood of $\bar{x}$. Now consider any smooth path $\gamma(t)(t \in \mathbf{R})$ in $\overline{\operatorname{Fix}(K)}$ with limit point $\gamma( \pm \infty)=\bar{x}$ which is tangent to the stable subspace for $t \rightarrow \infty$ and to the unstable subspace for $t \rightarrow-\infty$. In a neighbourhood of each point of the curve $\gamma$ the orbit space can be written as a product and so one can easily construct a vector field tangent to the curve and which extends to a stratified $C^{\infty}$ vector field tangent in that neighbourhood. Taking a partition of unity subordinate to a locally finite subcover, one constructs a $C^{\infty}$ vector field on the orbit space (vanishing outside a neighbourhood of the curve $\gamma$ ) and which has $\gamma(t)$ as a homoclinic trajectory based at $\bar{x}$. By the lifting theorem of Schwarz [10] this vector field can be lifted to a $C^{\infty} G$-equivariant vector field on $M$. Moreover the lift of $\gamma$ will contain an RHT based at $x$. It follows that $X_{3}$ is not empty.

Since $\operatorname{dim}\left(W^{u}(x) \cap \operatorname{Fix}(K)\right)+\operatorname{dim}\left(W^{s}(x) \cap \operatorname{Fix}(K)\right) \geqslant \operatorname{dim} \operatorname{Fix}(K)+1$ there is an open set $X_{4} \subset X_{3}$ of flows that have transverse intersection of these manifolds and hence the RHTs in the open set $X_{4}$ are robust as required. 
Remark 4.2. Notice that although $K \neq K^{g}$ we do not require that $H$ and $H^{g}$ differ for this result. The examples in Sections $1 \cdot 1$ and 1.2 have $H \neq H^{g}$ and $H=H^{g}$ respectively. Moreover the constructed RHTs may or may not be attractors.

Remark 4.3. Condition [HTL] is not necessary, but it simplifies the proof considerably. Any more general hypothesis on the representation of $H$ on $T_{x} M$ would suffice provided it allows construction of open sets of vector fields satisfying equation $(4 \cdot 1)$; see Section $4 \cdot 3$ for an example. It is worth noticing that [HTL] is satisfied whenever $H$ is abelian, which holds in almost all the examples in Section 3.

Remark 4.4. Condition [HTG] is obviously necessary, and requires knowledge of the group action in order to be verified. However, if the action is a complex or symplectic representation of a finite group then the symmetry-type sets (i.e. those with constant isotropy) are always connected, so that [HTG] is always verified; see Theorem $4 \cdot 6$.

\section{4·1. An example of a robust $\mathrm{RHT}$ with $S O(3)$ symmetry}

Theorem $4 \cdot 1$ can be applied to produce new examples of robust RHTs in a variety of contexts. For example, let $G=S O(3)$ and let $V_{1}$ be the 3-dimensional irreducible representation of $S O(3)\left(V_{1}=\mathbf{R}^{3}\right)$, and $V_{2}$ the 5-dimensional irreducible representation, consisting of trace-0 $3 \times 3$ real symmetric matrices (equivalently, the first and second order spherical harmonics respectively). Let $M=\left(V_{1} \oplus V_{2}\right) \oplus \mathbf{C}$. This is a 16-dimensional representation of $S O(3)$, isomorphic to the sum $\mathbf{C}^{3} \oplus V_{2}^{\mathrm{C}}$, where $V_{2}^{\mathrm{C}}$ consists of complex trace- 0 symmetric $3 \times 3$ matrices. $S O(3)$ acts on $V_{1}$ by multiplication on the left, and on $V_{2}$ by conjugation.

Note that action of $S O(3)$ on $V_{2}$ has non-trivial generic orbit type (equal to the conjugacy class of the subgroup $H$ defined below), since every symmetric matrix is diagonalizable. On the other hand, if the real and imaginary parts of a complex matrix in $V_{2}^{\mathrm{C}}$ have no common eigenvector then the isotropy of the point is trivial.

Denote by $R_{\theta}^{x}$ the rotation by $\theta$ about the $x$-axis. Let $H=\mathbf{D}_{2}$ be the group generated by $R_{\pi}^{x}$ and $R_{\pi}^{y}$. Then $\operatorname{Fix}(H)$ consists of diagonal matrices. Let $K=\left\langle R_{\pi}^{x}\right\rangle$, so that $\operatorname{Fix}(K)$ consists of certain block diagonal matrices, and let $g=R_{\pi / 2}^{y}$.

Theorem 4.5. Suppose $G=S O(3)$ and $M$ is the 16-dimensional representation as above. Then there exist robust RHTs on $M$ for vector fields with symmetry $G$.

Proof. We verify that the hypotheses of Theorem $4 \cdot 1$ are satisfied and then use the conclusion of that theorem. To see that $(K, g, H)$ is a homoclinic triple, note that $g$ does not fix $\operatorname{Fix}(K)$ as it maps the $x$ axis onto the $z$ axis, and observe that $H$ is generated by $K$ and $K^{g^{-1}}$. For [HTL] it suffices to point out that $N_{G}(K)=$ $N_{G}\left(K^{g^{-1}}\right)=O(2)$ (generated by all $R_{\theta}^{x}$ and $R_{\pi}^{y}$ ), and this contains $H$.

There remains to show that the global connectivity condition is satisfied, which can be achieved explicitly. Since $S O(3)$ acts independently on each summand in $M$, it follows that for any subgroup

$$
\operatorname{Fix}(K, M)=\operatorname{Fix}\left(K, V_{1}^{\mathrm{C}}\right) \oplus \operatorname{Fix}\left(K, V_{2}^{\mathrm{C}}\right),
$$

moreover, that $\operatorname{Fix}\left(K, V^{\mathrm{C}}\right)=\operatorname{Fix}(K, V)^{\mathrm{C}}$, and finally that the isotropy subgroup of $(u+i v, A+i B) \in M$ is the intersection of the isotropy subgroups of each of $u, v, A$ and $B$. 
Thus $\operatorname{Fix}(K, M)$ consists of points of the form

$$
\left[\left(\begin{array}{l}
t \\
\hline 0
\end{array}\right),\left(\begin{array}{l|l}
a & 0 \\
\hline 0 & A
\end{array}\right)\right]
$$

where $a, t \in \mathbf{C}$ and $A$ is a $2 \times 2$ complex symmetric matrix of $\operatorname{trace} \operatorname{tr}(A)=-a$. A typical point in $\operatorname{Fix}(H, M)$ is a point of the form $U=(0, \operatorname{diag}[\alpha, \beta, \gamma])$, and then $g \cdot U=(0, \operatorname{diag}[\gamma, \beta, \alpha])$. We need to show that there is a path in $\operatorname{Fix}(K, M)$ connecting $U$ and $g \cdot U$ consisting entirely of points with isotropy precisely $K$.

Now, $\operatorname{dim} \operatorname{Fix}(K, M)=8$, and $\operatorname{dim} \operatorname{Fix}(H)=4$. Furthermore $N_{G}(K) \simeq O(2)<$ $S O(3)$, so that the set of points in $\operatorname{Fix}(K, M)$ with orbit type $(H)$ is of dimension $4+1=5$ and so its complement in $\operatorname{Fix}(K)$ is connected. The only other points in $\operatorname{Fix}(K, M)$ with higher symmetry are those fixed by $S O(2)<N_{G}(K)$ (generated by rotations about the $x$-axis) which are just those of the form $(4 \cdot 2)$ with $A$ a multiple of the identity, which is a set of real dimension only 4 , and so again does not separate $\operatorname{Fix}(K, M)$.

\subsection{A class of finite groups admitting robust $\mathrm{RHTS}$}

The next result gives sufficient conditions on a finite group $G$ such that a representation $M$ of $G$ can be found that admits robust RHTs for $G$ equivariant vector fields on $M$.

Theorem 4·6. Suppose that $G$ is a finite group with a homoclinic triple $(K, g, H)$ satisfying in addition [HTL]. Let $M$ be the complex regular representation of $G$ (of real dimension $2|G|$ ). Then there is an open set of $G$-equivariant vector fields on $M$ for which there is an RHT with isotropy $K$ and twist $g$.

Proof. This follows from Theorem 4·1. Indeed, every subgroup of $G$ is an isotropy subgroup for this representation so that $K, K^{g^{-1}}$ and $H$ are isotropy subgroups as required, and hypothesis [HTL] is satisfied by assumption. Since all strata are even-dimensional there are no connectivity restrictions and we can satisfy [HTG].

Example 4.7. As an example of an application of this theorem, consider the eightelement dihedral group

$$
D_{4}=\left(\left\{\rho, \kappa: \rho^{4}=\kappa^{2}=1, \rho \kappa=\kappa \rho^{-1}\right\}\right) .
$$

This has a subgroup $K=\langle\kappa\rangle$ and element $g=\rho$ that satisfy the hypotheses of the theorem (in this case $H=\left\langle\kappa, \rho^{2}\right\rangle$ ) and so we can construct robust cycles with $\mathbf{D}_{4}$ symmetry for a flow on $\mathbf{R}^{16}$. This is identical in form to the Kuramoto-Sivashinsky example in Section 1.2 but in a much higher dimensional space.

Example $4 \cdot 8$. Similarly, one can use Theorem $4 \cdot 6$ to show that the group occurring in the Guckenheimer-Holmes example in Section 1.1 permits robust RHTs. Namely, let $G=\mathbf{Z}_{2} \prec S_{3}$, see Section $3 \cdot 3$ and consider $K=\langle\kappa\rangle$ and $g=\rho$. Again, this produces a much higher dimensional space than the original example in $\mathbf{R}^{3}$.

Example 4.9. The homoclinic triples for wreath products defined in Example $3 \cdot 6$ with $\mathscr{L}$ finite satisfy the hypothesis [HTL] of Theorem $4 \cdot 1$. There are therefore open sets of equivariant vector fields on the complex regular representation with RHTs for which these triples occur. 
Table 1. Character table for $\mathbf{D}_{8}$. The final two columns give the corresponding representations of $\mathbf{D}_{4}=\left\langle\kappa, \rho^{2}\right\rangle$ and $\mathbf{Z}_{2}=\langle\kappa\rangle$ via restriction (the characters for the $\mathbf{D}_{4}$ and $\mathbf{Z}_{2}$ representations can be deduced from this table).

\begin{tabular}{rrrrrrrrrl}
\hline & $e$ & $(\kappa)$ & $(\kappa \rho)$ & $(\rho)$ & $\left(\rho^{2}\right)$ & $\left(\rho^{3}\right)$ & $\rho^{4}$ & $\mathbf{D}_{4}$ & $\mathbf{Z}_{2}$ \\
\hline$A_{0}$ & 1 & 1 & 1 & 1 & 1 & 1 & 1 & $A_{0}^{\prime}$ & $A_{0}^{\prime \prime}$ \\
$A_{1}$ & 1 & -1 & -1 & 1 & 1 & 1 & 1 & $A_{1}^{\prime}$ & $A_{1}^{\prime \prime}$ \\
$A_{2}$ & 1 & 1 & -1 & -1 & 1 & -1 & 1 & $A_{0}^{\prime}$ & $A_{0}^{\prime \prime}$ \\
$A_{3}$ & 1 & -1 & 1 & -1 & 1 & -1 & 1 & $A_{1}^{\prime}$ & $A_{1}^{\prime \prime}$ \\
\hline$E_{1}$ & 2 & 0 & 0 & $\sqrt{2}$ & 0 & $-\sqrt{2}$ & 0 & $E^{\prime}$ & $A_{0}^{\prime \prime}+A_{1}^{\prime \prime}$ \\
$E_{2}$ & 2 & 0 & 0 & $-\sqrt{2}$ & 0 & $\sqrt{2}$ & 0 & $E^{\prime}$ & $A_{0}^{\prime \prime}+A_{1}^{\prime \prime}$ \\
$F$ & 2 & 0 & 0 & 0 & -2 & 0 & 2 & $A_{2}^{\prime}+A_{3}^{\prime}$ & $A_{0}^{\prime \prime}+A_{1}^{\prime \prime}$ \\
\hline
\end{tabular}

Remark 4.10. Many examples of robust RHTs in the literature are for irreducible representations (for example, the Guckenheimer-Holmes example in Section 1·1) or for group actions where there are only a few irreducible components (for example, the Kuramoto-Sivashinsky example in Section 1.2 where there are two). Our result, Theorem $4 \cdot 6$, by contrast gives robust RHTs on much larger dimensional spaces with many irreducible components. Usually, no irreducible representation will be sufficiently rich to satisfy the hypothesis of Theorem $4 \cdot 1$, and this begs the question of how to obtain optimally small estimates for the dimension of the group action one needs to consider in order to obtain robust RHTs for a given group.

Remark $4 \cdot 11$. If $G$ is a finite group that satisfies Theorem $4 \cdot 6$ we say that $G$ is robust. Clearly any finite group that has a robust subgroup is robust, giving another way of constructing many groups admitting RHTs.

\subsection{An example with $G=\mathbf{D}_{8}$}

We present an example demonstrating the difference between the necessary conditions of Proposition $2 \cdot 3$ and the sufficient conditions of Theorem $4 \cdot 1$. It is in some sense the simplest example of a homoclinic triple that does not satisfy the extra sufficient condition [HTL] of Theorem $4 \cdot 1$, and appears in the $\mathbf{D}_{8}$-table in Section $3 \cdot 1$. We consider complex representations so that the global connectivity hypothesis [HTG] of Theorem $4 \cdot 1$ is automatically satisfied, as in Theorem $4 \cdot 6$.

Using the notation for the dihedral groups introduced in Section $3 \cdot 1$, consider the homoclinic triple $(K, g, H)$, where $K=\langle\kappa\rangle, g=\rho$ and $H=\left\langle\kappa, \rho^{2}\right\rangle$. This is a homoclinic triple by Theorem $3 \cdot 5$, and indeed it is a minimal triple.

Consider the irreducible representations of $G=\mathbf{D}_{8}$, of which there are seven in all (Table 1). It can be seen from the table that, for a representation $V$ of $\mathbf{D}_{8}, H$ is an isotropy subgroup if and only if $V$ contains at least one copy of $A_{2}$, and $K$ is an isotropy subgroup if and only if $V$ contains at least one copy of either $E_{1}$ or $E_{2}$.

Theorem 4.12. Let $V$ be a complex representation of $\mathbf{D}_{8}$ for which $K$ and $H$ are isotropy subgroups, where $K=\mathbf{Z}_{2}$ and $H=\mathbf{D}_{4}$ as above. There is an open set of equivariant vector fields with homoclinic triple $(K, g, H)$ with $g=\rho$ if and only if $V$ contains at least one copy of the irreducible representation F (see Table 1).

Proof. This is based on the proof of Theorem $4 \cdot 1$ and Remark $4 \cdot 3$. Let $x \in \operatorname{Fix}(H)$. 
Then $T_{x} V=V$ and the linearization $L$ of the equivariant vector field at $x$ is $H$ equivariant, and we need to consider the eigenvalues of $L$ on each irreducible representation. Consider the isotypic decomposition of $V$ :

$$
V=a_{0} A_{0} \oplus a_{1} A_{1} \oplus a_{2} A_{2} \oplus a_{3} A_{3} \oplus e_{1} E_{1} \oplus e_{2} E_{2} \oplus f F,
$$

where $a_{0}, \ldots, f$ are non-negative integers. Then

$$
\begin{aligned}
\operatorname{Fix}(H, V) & =a_{0} A_{0} \oplus a_{2} A_{2} \\
\operatorname{Fix}(K, V) & =a_{0} A_{0} \oplus a_{2} A_{2} \oplus e_{1} E_{1}^{(s)} \oplus e_{2} E_{2}^{(s)} \oplus f A_{2}^{\prime} \\
\operatorname{Fix}\left(K^{g^{-1}}, V\right) & =a_{0} A_{0} \oplus a_{2} A_{2} \oplus e_{1} E_{1}^{(d)} \oplus e_{2} E_{2}^{(d)} \oplus f A_{3}^{\prime} .
\end{aligned}
$$

Some of this notation needs explaining. The $A_{k}$ parts should be self-explanatory. The two representations $E_{1}$ and $E_{2}$ of $\mathbf{D}_{8}$ are the usual symmetry groups of the regular octagon; in the first $\rho$ acts by rotation by $\pi / 4$ while in $E_{2}$ it acts by rotation by $3 \pi / 4$. Restricting the action to $H$, picks out a square in the octagon, whose vertices lie at alternating vertices of the octagon say, and then $E_{j}^{(s)}$ is a line of reflection in $E_{j}$ passing through midpoints of a pair of sides of the square, while $E_{j}^{(d)}$ is a diagonal line of the square. Note that any $H$-equivariant linear vector field on $E_{j}^{\prime}$ has the same eigenvalues on both $E_{j}^{(s)}$ and $E_{j}^{(d)}$, so if $f=0$ in the representation, the eigenvalues on $\operatorname{Fix}\left(K^{g^{-1}}, V\right)$ and $\operatorname{Fix}(K, V)$ cannot be distinct.

On the other hand, the irreducible representation $F$ decomposes into two $H$ irreducibles, $F=A_{2}^{\prime}+A_{3}^{\prime}$. Indeed, in the action of $\mathbf{D}_{8}$ on $F, \rho$ acts as rotation by $\pi / 2$, so that $\mathbf{D}_{8}$ acts as the symmetry group of the square, and the action of $H$ is just by a pair of reflections. These two reflections are in fact those in $K$ and $K^{g^{-1}}$, and so $\operatorname{Fix}(K, F)=A_{2}^{\prime}$ say, and $\operatorname{Fix}\left(K^{g^{-1}}, F\right)=A_{3}^{\prime}$. It follows that an $H$-equivariant $L$ can be chosen so that the eigenvalues on $A_{2}^{\prime}$ and $A_{3}^{\prime}$ are of opposite sign, and there is an open set of such $L$. Consequently, if $f \geqslant 1$ then there is an open set of linear vector fields $L$ satisfying $(4 \cdot 1)$, as required by Remark $4 \cdot 3$.

\section{Discussion}

There has been much work on robust heteroclinic cycles that has looked at structure and existence of RHTs for given group representations. What we have attempted here is to understand better the group-theoretic conditions on a group necessary to find a representation admitting RHTs. We have found necessary conditions in Proposition $2 \cdot 3$ and sufficient conditions in Theorem $4 \cdot 1$ but there remains a gap in the hypotheses that would be nice to close. Specifically one would like to be able to characterize a weaker version of [HTL] that would be both necessary and sufficient, and Remark $4 \cdot 3$ together with the example in Section $4 \cdot 3$ shows that this condition must include some information on the local structure of the action.

A number of other questions are suggested by this study. As mentioned already, there are optimality questions; for example, given a group with a homoclinic triple that does admit robust RHTS, how small a representation can one consider to find a robust RHT? Also, how rare are robust RHTs for equivariant systems? For example, which finite groups of order $n$ have the necessary complexity to admit robust RHTs? Which irreducible representations of finite groups of dimension $n$ admit robust RHTs?

It does not seem to be a trivial task to extend or generalize the results here to apply to robust heteroclinic cycles, due partly to the fact that the interconnection 
possibilities are much greater, and ensuring their robustness requires that many local and global conditions are fulfilled simultaneously (see for example [8]). However, it should be possible to generalize the results to apply to homoclinic cycles between classes of more general transitive invariant sets.

\section{REFERENCES}

[1] A. P. S. Dias, B. Dionne and I. Stewart. Heteroclinic cycles and wreath product symmetries. Dynamics and Stability of Systems 15 (2000), 353-385.

$[2]$ B. Dionne, M. Golubitsky and I. Stewart. Coupled cells with internal symmetry: I. Wreath products. Nonlinearity 9 (1996), 559-574.

[3] M. FiELD. Symmetry breaking for compact Lie groups, Mem. AMS 574 (1996).

[4] M. Field. Lectures on Dynamics, Bifurcation and Symmetry. Pitman Research Notes in Mathematics 356 (1996).

[5] J. Guckenheimer and P. Holmes. Structurally stable heteroclinic cycles. Math. Proc. Camb. Phil. Soc. 103 (1988), 189-192.

[6] I. G. Kevrekidis, B. Nicolaenko and J. C. Scovel. Back in the saddle again: a computer assisted study of the Kuramoto-Sivashinsky equation. SIAM J. Appl. Math. 50 (1990), $760-790$.

[7] M. Krupa and I. Melbourne. Asymptotic stability of heteroclinic eycles in systems with symmetry. Erg. Th. Dyn. Sys. 15 (1995), 121-147.

[8] I. Melbourne, P. Chossat and M. Golubitsky. Heteroclinic cycles involving periodic solutions in mode interactions with $O(3)$ symmetry. Proc. Roy. Soc. Edinburgh 113A (1989), $315-345$.

[9] I. Melbourne, M. Dellnitz and M. Golubitsky. The structure of symmetric attractors. Arch. Rational Mech. Anal. 123 (1993), 75-98.

[10] G. Schwarz. Lifting smooth homotopies of orbit spaces. Publ. Math. Inst. Hautes Etudes Sci. $51(1980), 37-135$. 